\title{
OPEN Sacrificial gold coating enhances transport of liquid metal in pressurized fountain pen lithography
}

\author{
Gideon I. Livshits ${ }^{1,2 \bowtie}$, Jiannan Bao ${ }^{1}$, Leo Sakamoto ${ }^{1}$, Tomoki Misaka ${ }^{1}$, Yuki Usami ${ }^{1}$, \\ Yoichi Otsuka $^{1} \&$ Takuya Matsumoto ${ }^{1 凶}$
}

Liquid metals have attracted attention as functional components for moldable electronics, such as soft flexible connectors, wires or conductive ink. The relatively high surface tension $\left(>400 \mathrm{mN} \mathrm{m}^{-1}\right)$ and the fact that liquid metals do not readily wet ceramic or oxide surfaces have led to devising unique techniques to spread the liquid and mold its shape. These techniques include surface modification, electrowetting and vacuum filling of channels. This work presents an injection technique based on pressurized fountain pen lithography with glass nanopipettes developed to directly pattern liquid metal on flat hard substrates. The liquid metals were eutectic alloys of Gallium, including GalliumIndium (EGaln), Gallium-Indium-Zinc and Gallium-Indium-Tin. The nanopipettes were coated internally with gold, acting as a sacrificial layer and facilitating the wetting of the pipette down to its pore, with an inner diameter of $~ 100-300 \mathrm{~nm}$. By applying hydrodynamic pressure to the connected end of the pipette, the metal was extruded through the pore, forming long continuous ( $>3 \mathrm{~mm}$ ) and narrow $(\sim 1-15 \mu \mathrm{m})$ metal lines on silicon oxide and gold surfaces at room temperature and ambient conditions. With this robust platform, it is possible to pattern liquid metals on a variety of substrates and geometries down to the micron range.

Various methodologies of direct writing of liquid metal shapes and functional components have been described in the scientific literature ${ }^{1-10}$. In particular, Ladd et al. ${ }^{5}$ demonstrated $3 \mathrm{D}$ printing of free-standing liquid metal microstructures of varying sizes, and Yoon et al. ${ }^{2}$ demonstrated a platform for direct writing of liquid metal patterns, 70-80 $\mu \mathrm{m}$ wide, on uneven or curved surfaces. The interest in such techniques lies in their top-down control over architecture, enabling the formation of $2 \mathrm{D}$ or $3 \mathrm{D}$ electrically conductive structures without the need for sacrificial masking steps, which are routinely employed in standard lithographic techniques. The main challenge in developing a reproducible and controllable platform for this type of lithography is the transition from the macro-scale to the micro or nano regime due to the native high surface tension of liquid metals ${ }^{11,12}$, which makes it very difficult to form well-defined micro/nano-structures. Systematic improvements in pattern resolution were made by Boley et al. ${ }^{1}$ who demonstrated writing lines with various widths from $535 \mu \mathrm{m}$ down to $83 \mu \mathrm{m}$, and more recently, by Shin et al. ${ }^{13}$, who reported printing lines with a width as small as $22 \mu \mathrm{m}$ on flat, inclined and curved substrates. In the latter study, the improved resolution was mainly due to sensor-control of the distance between the substrate and the tip of the nozzle in the vertical direction. This enabled the authors to utilize metallic nozzles with an inner diameter that was 3-5 times the width of the drawn line. Park et al. ${ }^{9}$ have taken this technique a step further, by utilizing glass pipettes instead of metallic nozzles, with inner diameters ranging from 5 to $40 \mu \mathrm{m}$. By controlling the tip-substrate distance, they were able to obtain controlled pattern features with a minimal width of just under $2 \mu \mathrm{m}$. In the present work, we address this challenge further by proposing to utilize glass nanopipettes to control the pore size down to the $0.1 \mu \mathrm{m}$ scale.

Working with open-ended nanopipettes requires much higher pressures to overcome the surface tension of the liquid material inside microscale apertures ${ }^{9}$. When properly configured, as we shall demonstrate in this manuscript, the resulting patterns can be reduced to a width of $1 \mu \mathrm{m}$. In order to drive the metal liquid into the nanopipette, we experimented initially with several methodologies, such as electrowetting using an

${ }^{1}$ Department of Chemistry, Graduate School of Science, Osaka University, 1-1 Machikaneyama-cho, Toyonaka, Osaka 560-0043, Japan. 'Present address: Lev Academic Center, Jerusalem College of Technology, Havaad HaLeumi St. 21, Jerusalem 9116001, Israel. ${ }^{\square}$ email: livshits.gideon@mail.huji.ac.il; matsumoto-t@ chem.sci.osaka-u.ac.jp 


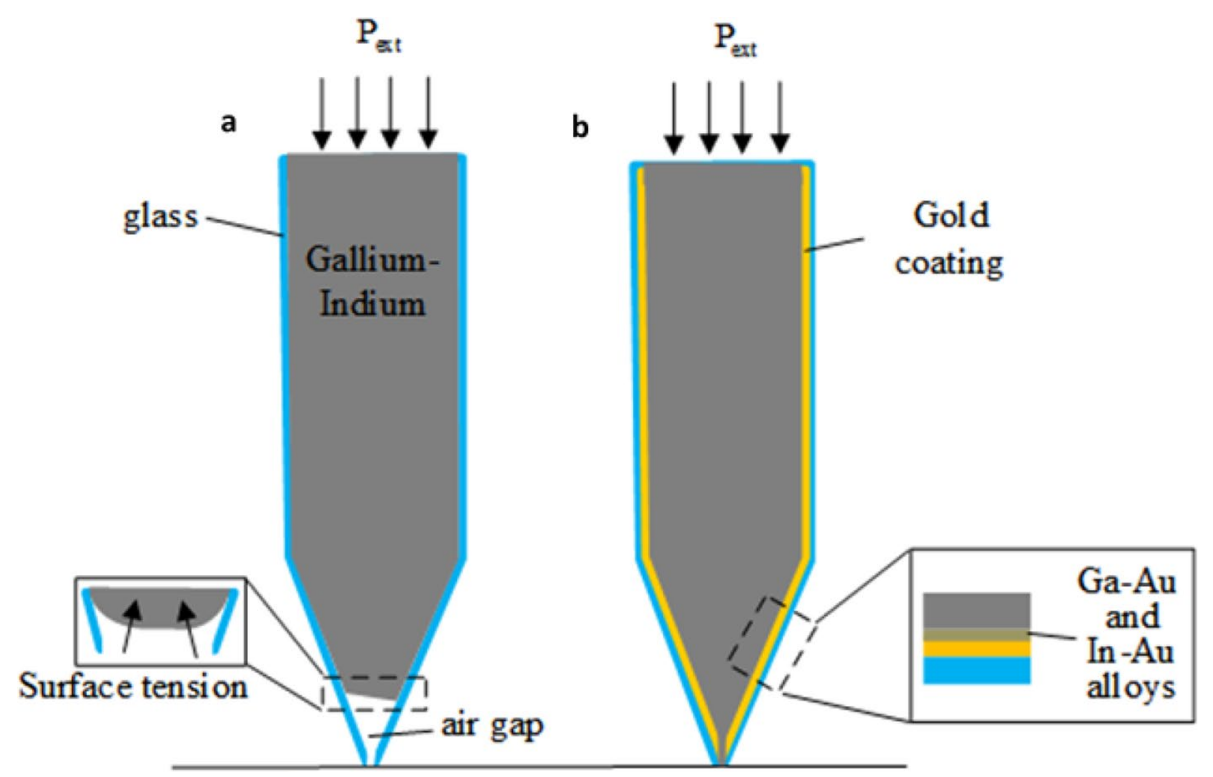

Figure 1. Experimental Scheme. (a) When external pressure is applied to the liquid EGaIn inside the glass pipette, it proves insufficient in overcoming the surface tension, leaving a gap of air, beyond which it would not fill. Continuing to apply the pressure beyond this point typically results in the explosive shattering of the glass pipette. (b) When the glass is coated with gold from the inside, the molecular interactions between the gold coating and the liquid metal enable wetting under pressure. As the pressure increases, the liquid fills up to the pore, while the gold layer is absorbed almost immediately into the metal liquid.

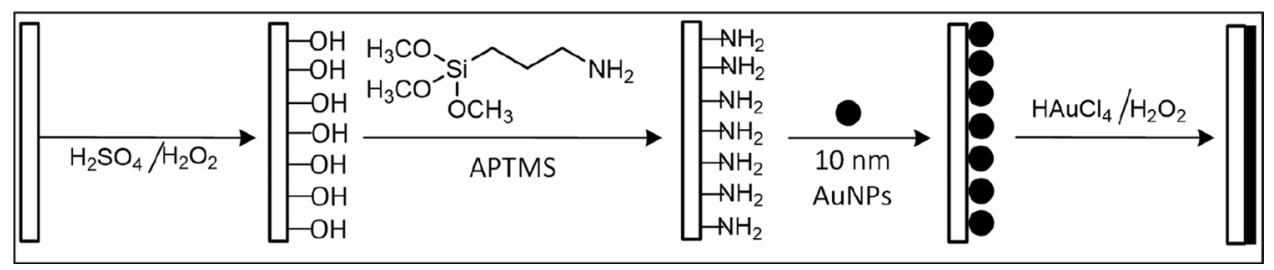

Figure 2. Scheme of the chemistry of the gold coating of glass. The illustration is adapted from Hu et al. ${ }^{23} \mathrm{Left}$ to right: after cleaning and surface activation, the inner surface of the glass tubes was functionalized with a solution of APTMS overnight by pumping the solution directly through the tubes. After thoroughly cleaning the tubes from the inside, they were seeded with $10 \mathrm{~nm}$ GNPs for 3-4 h, and electroless deposition was activated by introducing a solution of Gold (III) chloride trihydrate and $\mathrm{H}_{2} \mathrm{O}_{2}$ as a reducing agent. The reaction commenced as soon as the solution reached the cavity and was allowed to continue for $20 \mathrm{~min}$. The procedure is demonstrated in Fig. S1 with more details in the "Methods" section.

adjacent counter-electrode ${ }^{14-16}$ and heating-induced spreading and wetting using as a heating element a coil wrapped around the pipette ${ }^{17}$. These techniques showed limited promise and proved too weak (even at $\sim 10 \mathrm{kV}$ and $\sim 400{ }^{\circ} \mathrm{C}$, respectively) to overcome the glass-metal wetting barrier to manipulate EGaIn through the submicron aperture of the pipette. To address this challenge, we opted instead for chemical or reactive wetting ${ }^{12}$, by exploiting the affinity of both Gallium and Indium to form metal alloying compounds with gold ${ }^{18-22}$. A schematic representation of the experiment is shown in Fig. 1. When the metal liquid is introduced into a pristine uncoated glass pipette (Fig. 1a), it forms a boundary due to its surface tension, and an air gap is formed between this boundary and the pore. It is possible to apply pressure to the liquid metal to force it farther into the tube, but at a certain maximum pressure the tube would shatter. In order to reduce the needed pressure and facilitate the filling process by effectively breaking the boundary, we induce wetting at the glass walls by coating the inner surface of the glass capillary with gold (Fig. 1b). It is the alloying reactions around the inner surface that break the boundary or at least make it unstable enough, so that the thin gold layer is consumed by EGaIn, and the pressure that is required to fill the tube is significantly reduced.

\section{Results}

In order to obtain a smooth and uniform coating of the glass nanopipette, prior to its fabrication, the glass capillary was coated with gold from the inside using electroless deposition following published protocols ${ }^{23,24}$ with several key modifications, depicted schematically in Fig. 2. Most notably, surface functionalization with 


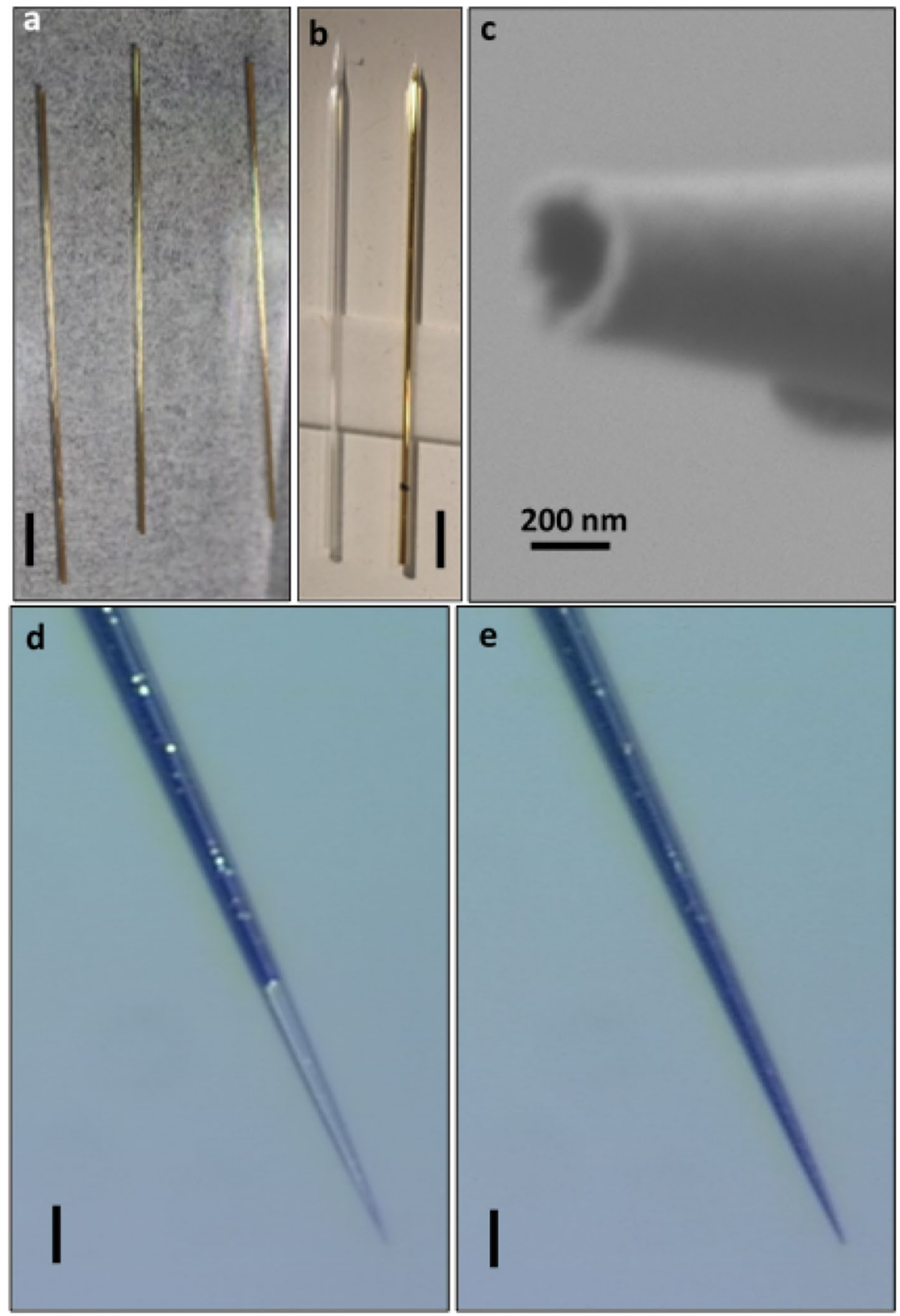

Figure 3. Pipette coating and filling. (a) Optical image of three gold-coated glass capillaries. The coating is only from the inside; it is continuous and conductive. Scale bar is $10 \mathrm{~mm}$. (b) Optical image of two nanopipettes, bare (left) and coated (right), for comparison. The coating becomes sparser towards the aperture due to the thermal extrusion process. Scale bar is $5 \mathrm{~mm}$. (c) SEM micrograph of a tip of a coated pipette as in (b), formed under thermal extrusion. (d,e) Optical images of a coated nanopipette, at the initial, (d), and final, (e), stages of filling with EGaIn, respectively. Scale bar is $20 \mu \mathrm{m}$. Traces of the coating are barely visible, having been dissolved inside the EGaIn. To completely fill the pipette requires $\sim 15-25 \mathrm{~atm}$. Once the pipette is filled, the pressure is dropped, and the pipette remains full. A movie of the filling process is available as Supplementary Information S1.

(3-Aminopropyl)trimethoxysilane (APTMS), self-assembly of the gold nanoparticles (GNPs) and the subsequent reduction of the gold salt were all done by pumping the solutions directly through the glass capillaries, thereby ensuring the localization of the reaction to the inner surface of the capillaries. Fig. S1 in the Supplementary Information shows the experimental setup with the connected tubes at the final stages of the reaction, as the color inside changes from dark red to gold. A complete description of the chemical synthesis is given in the 


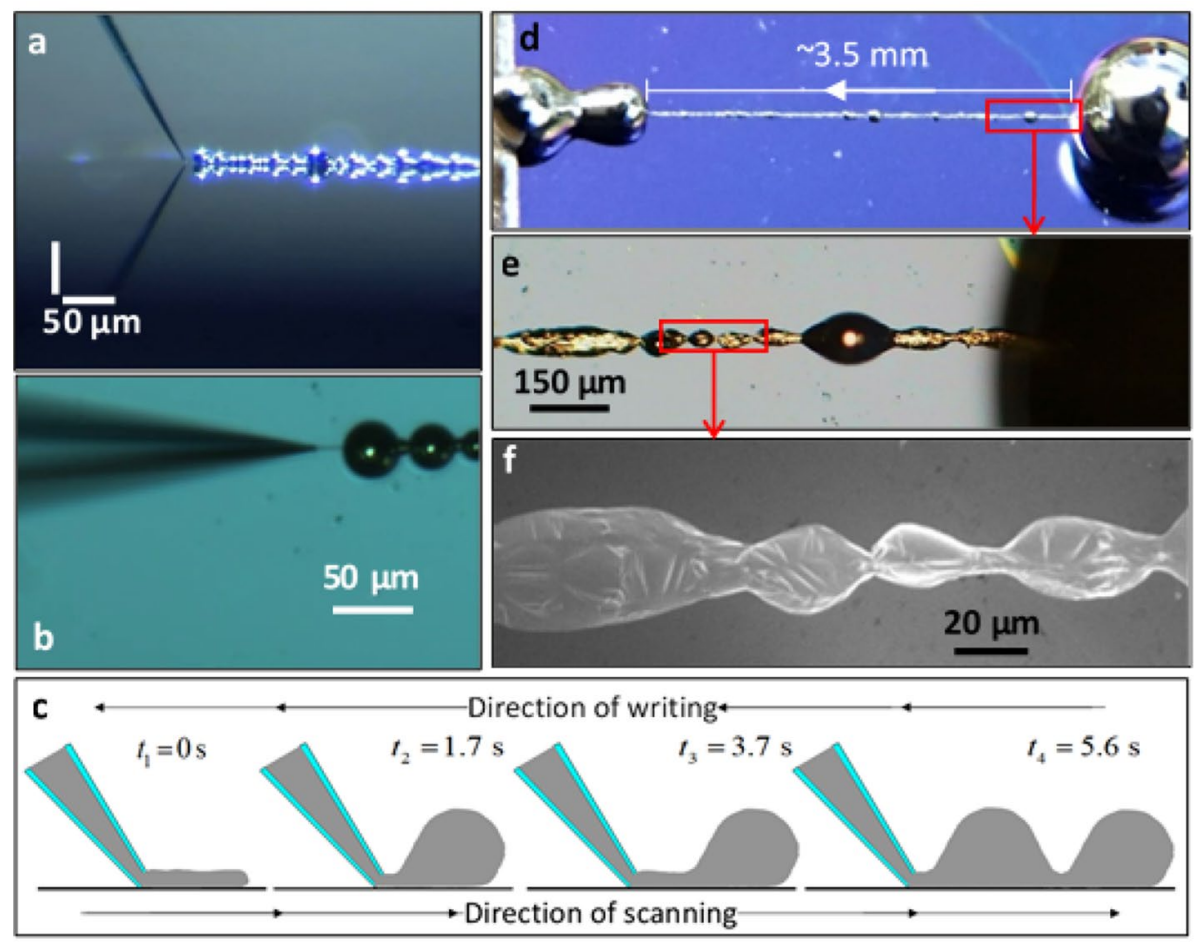

Figure 4. EGaIn on $\mathrm{SiO}_{2}$. (a,b) Optical images (side and top view, respectively) of the process of extrusion (under constant pressure, $\sim 45 \mathrm{~atm}$ ) of EGaIn on a hydroxilated silicon surface. Microdroplets of the EGaln are apparent. (c) A schematic illustration of the process (left to right): after a short segment of the line is released onto the surface, typically $15-20 \mu \mathrm{m}$ long and $1-3 \mu \mathrm{m}$ wide, the line quickly congeals (after 1.7-1.9 s) into a microdroplet. As the substrate is being moved in the opposite direction, the metal is continuously extruded, a second line is formed, and then again congeals. In this way, a series of joined microdroplets is formed on the surface. See Supplementary Information S2 for a movie of this dynamic deposition. (d) Optical image of the completed pattern, extending for over $3.5 \mathrm{~mm}$. (e) Optical image of the marked region in (d), showing the area near the initial release (right). (f) Electron micrograph of the marked region in (e), demonstrating in higher resolution the local structure. The droplets are deformed ellipsoids of varying lengths and widths. The initial stage of the line formation is shown in detail in the Supplementary Information, Fig. S2.

"Methods" section. The resulting coverage, shown in Fig. 3a, is uniform and adheres well to the glass with an estimated thickness of $\sim 100-300 \mathrm{~nm}$.

Nanopipettes were formed from coated capillaries using two different pulling techniques, one by thermal heating, and the other by localized laser heating. Although laser heating can control the formation of the cone shape and the aperture size independently, the resulting tips proved more brittle as they broke down under an applied pressure of $\sim 60 \mathrm{~atm}$. In contrast, the thermally-heated nanopipettes could withstand pressures exceeding $~ 100 \mathrm{~atm}$. This behavior may be attributed to non-uniform thermal relaxation of the glass during rapid localized laser heating as compared with the slower radiative and convective heating involved in the thermal coil heater. Therefore, in this study, only thermally-pulled pipettes are presented. From a single coated capillary two nanopipettes were formed by inserting the capillary into a tungsten thermal heater with preset values for all parameters, including the power, weight and heating steps, adjusted to produce a pore size of the inner diameter that is typically 100-300 nm (Fig. 3b,c). See "Methods" section for details. The coated pipette was then filled with EGaIn, and the application of external water pressure resulted in the metal filling the entire volume of the pipette down to its pore (Fig. 3d,e) without clogging by surface tension. A movie of the complete filling process is given in the Supplementary Information.

The nanopipette was then brought into contact with the underlying surface, and was kept stationary as the tip was being raster scanned against the moving substrate, at a typical rate of $10 \mu \mathrm{m} \mathrm{s}^{-1}$. Directly after the scanning commenced, pressure was applied to the top of the nanopipette at a constant flow rate, typically between 0.001 and $0.01 \mathrm{ml} \mathrm{min} \mathrm{m}^{-1}$. The buildup of pressure at the pore would increase precipitously until the visible eruption of a liquid metal drop on the surface, typically appearing at the range of 40-60 atm. Immediately following the eruption of the liquid, the external pressure was terminated, but the actual pressure difference would remain steady, dropping only slightly (by about $2-3 \%$ ). This pressure was observed to be constant, and the scanning would continue uninterrupted, all the while the metal liquid was flowing under this pressure difference with no additional external pressure. The scanning motion enabled the formation of continuous patterns extending for several millimeters (Figs. 4, 5 and 6). 

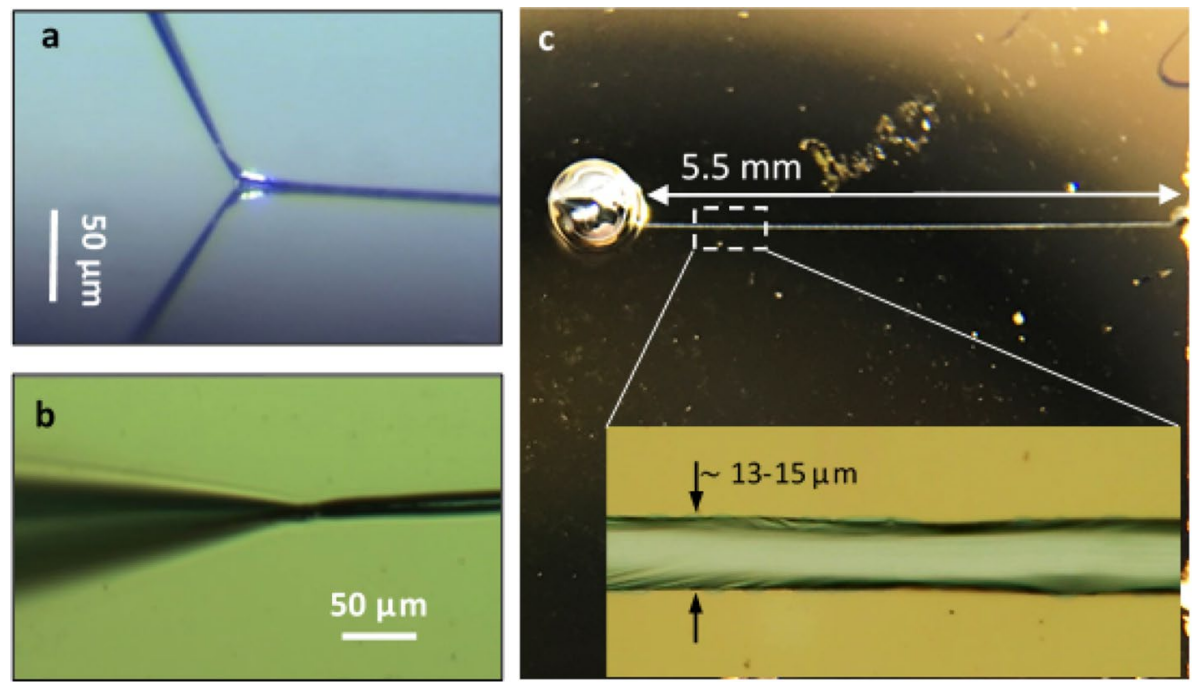

Figure 5. EGaIn on Au. (a,b) Optical images (side and top view, respectively) of the process of extrusion (under constant pressure, $\sim 55 \mathrm{~atm}$ ) of EGaIn on a gold surface. Once the initial droplet appears, the metal line that emerges is continuous. The entire deposition took $10 \mathrm{~min}$. See Supplementary Information S3 for a complete movie of the deposition. (c) Optical image of the completed pattern taken immediately after deposition. The inset is an optical image at higher resolution of the marked area in the main image, taken directly after patterning, showing a very clean deposition with sharp boundaries. Line width is $13-15 \mu \mathrm{m}$.
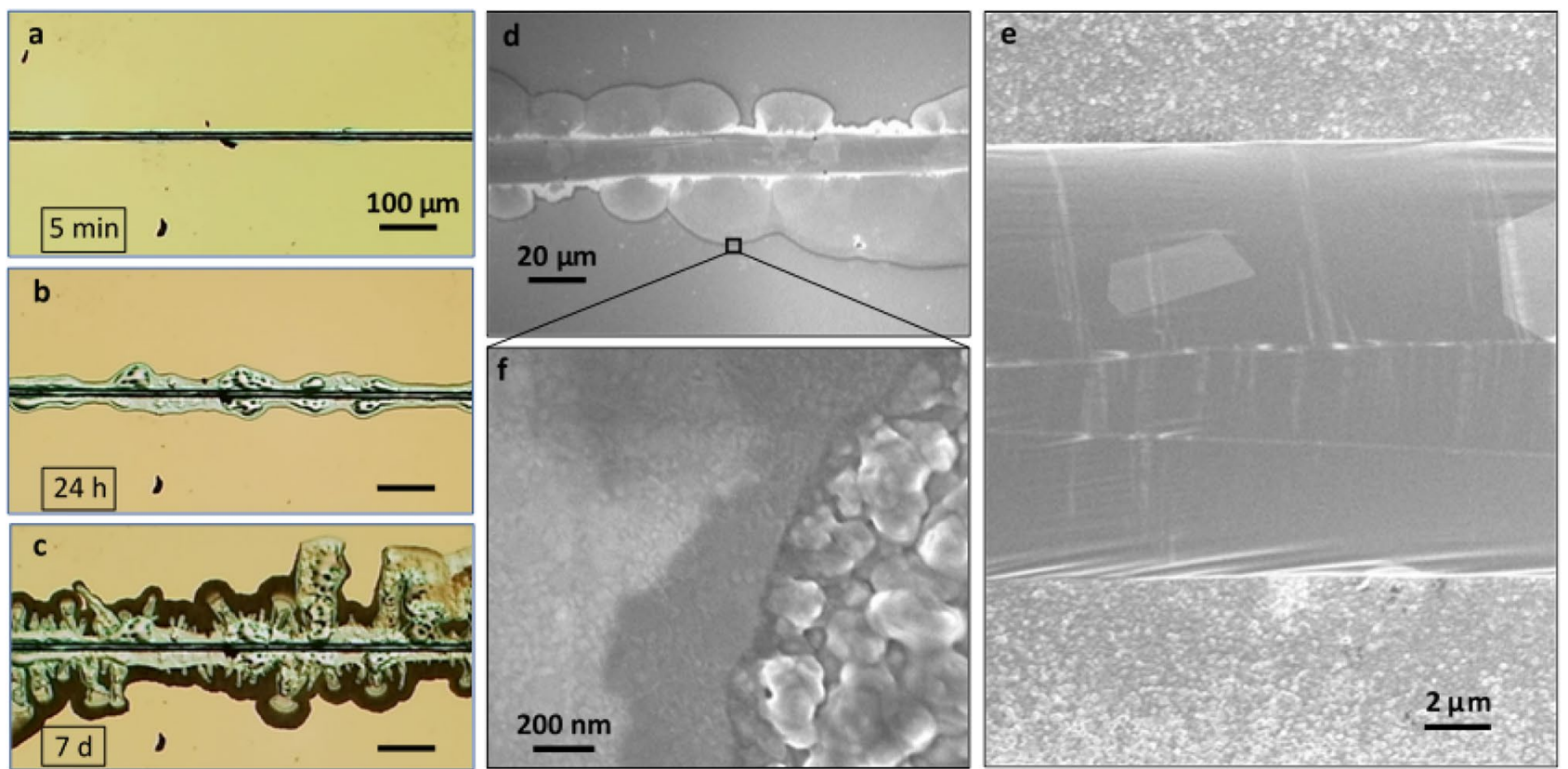

Figure 6. Surface spreading and reactivity. (a-c) Optical microscopy images of a segment of the patterned line in Fig. 5. The sample was kept inside a clean container at room temperature for several weeks. Image (a) was taken immediately after patterning showing a clear line, while (b) and (c) were taken $24 \mathrm{~h}$ and 7 days after patterning, respectively. The gradual spreading of the fronts is clearly visible as Ga and In form alloys with Au. (d-f) are electron micrographs of the pattern in (b). Large crystals are observed inside the line itself (e). Higher magnification of the front in (f) reveals different crystallite formation, varying in size as a function of the distance from the main source, from larger Ga-rich to smaller ones until the polycrystalline gold coating is reached. 
Figure 4 shows the result of drawing EGaIn on a silicon oxide surface. As the metal is extruded through the aperture (Fig. 4a,b), it expands by an order of magnitude, from an inner diameter of roughly 100-300 $\mathrm{nm}$ to a width on the surface of $1-3 \mu \mathrm{m}$. This printed line is unstable, and when a segment of the extruded metal reaches a length of $15-20 \mu \mathrm{m}$ (after $\sim 1.7 \mathrm{~s}$ ), the extruded material seems to spontaneously coalesce into a perfect droplet. This is a delayed reaction of the metal to the underlying ceramic surface and to the continual infusion of pressurized metal from the tip. It should be noted that since the pressure gradient is driving the extrusion, it is necessary to choose a scanning rate that would respond to this change and allow a sufficiently quick release of liquid metal. In this respect, it was found that slow rates (e.g., decreasing the rate from 10 to $2 \mu \mathrm{m} \mathrm{s}^{-1}$ ) produced very large globules of metal that coalesced into even bigger droplets of diameters $>100 \mu \mathrm{m}$.

During the translation of the substrate, the metal line continued to form continuously in the opposite direction, and so the formation of the droplets repeated itself. The resulting pattern appeared to consist of connected microdroplets. This process is illustrated in Fig. 4c. Since the lithography set-up is carried out at room temperature in ambient conditions, it is reasonable to assume that a passivation layer of oxide ${ }^{6,25}$ is formed as the metal is extruded, and that this layer eventually grows to cover the entire network of droplets. Optical and electron imaging (Fig. 4d-f) taken directly after deposition (Fig. 4d) and a day later (Fig. 4e,f and Fig. S2) show more complicated structures. The stable line appears to consist of a continuous network of elongated ellipsoids, with shorter narrow segments $(\sim 5 \mu \mathrm{m}$ wide) connecting bigger ones. These deformations of the perfect spherical shape could be attributed to the interplay between the surface tension of the liquid and the elastic properties of the oxide film that covers the liquid, suggesting that the adhesion of EGaIn to the $\mathrm{SiO}_{2}$ surface is not strong enough to maintain line shape opposing surface tension. A movie of the direct writing of the line is provided in the Supplementary Information.

To enhance the adhesion of EGaIn to the substrate surface, we capitalized on the observed alloying reaction of EGaIn to $\mathrm{Au}$, and introduced an Au layer on the hydroxylated Si substrate. Figure 5 shows the result of drawing EGaIn on a gold surface, which was prepared by thermal vapor deposition of $\sim 100 \mathrm{~nm} \mathrm{Au}$ on an $\mathrm{SiO}_{2} / \mathrm{Si}_{\text {wafer }}$ with $\mathrm{Cr}$ as an adhesion layer. In this case, a continuous line is drawn, without any condensation or droplet formation (Fig. 5a-c). The line is markedly wider than the line on the silicon oxide surface, with a width of $10-15 \mu \mathrm{m}$ (inset in Fig. 5c), possibly the result of the ensuant chemical reactions between the underlying gold substrate and the liquid metal. Figure 6 presents a comparison of the same line segment over the course of several days, with Fig. 6a taken directly after the pattern was formed. After $24 \mathrm{~h}$ (Fig. 6b), the spreading fronts on either side have exceeded the width of the original line, the outline of which is clearly visible. By that time, the line was coated by a passivating metal oxide layer, although the active reaction with the underlying gold, even after 7 days (Fig. 6c), suggests that liquid metal can still flow and diffuse into the gold region far beyond the contact area. Figure $6 \mathrm{~d}-\mathrm{f}$ show electron micrographs at higher resolution of the metal line and its surroundings, revealing the formation of several distinct crystallites of varying sizes.

We discovered that the diffusion of EGaIn on the gold layers depended on the thickness of the gold. By examining the reaction to the deposition of EGaIn on three substrates, with nominal gold thicknesses of $10 \mathrm{~nm}$ (Fig. S3), $40 \mathrm{~nm}$ (Fig. S4) and $100 \mathrm{~nm}$ (Figs. 5 and 6), we observed significant diffusion for $40 \mathrm{~nm}$ and $100 \mathrm{~nm}$, and no diffusion for $10 \mathrm{~nm}$. For the thicker layers $(>40 \mathrm{~nm})$, the diffusion behavior of In differed from that of $\mathrm{Ga}$ in the Au layer. Gallium diffusion into the gold appears as chemical wave-like behavior (Fig. S4g), while Indium seems to slowly disintegrate into smaller particles (Fig. S4h). For the thin layer $(10 \mathrm{~nm})$, the absence of visible diffusion suggests that after the thin layer of gold directly below the EGaIn had been consumed, there was not enough gold to accommodate lateral diffusion to the sides.

We examined pattern formation for two more eutectic GaIn alloys on gold, GaInSn and GaInZn (see Supplementary Information, Fig. S5). GaInSn produced thicker lines, 2.5-3 times wider than EGaIn (Fig. S5a,b), whereas GaInZn did not seem to wet the gold even after treatment with $\mathrm{HCl}$, producing large disconnected globules (Fig. S5c). This suggests that an oxide film forms more rapidly on GaInZn than on EGaIn, perhaps due to the oxidation propensity of metallic $\mathrm{Zn}^{26}$, thereby disrupting its chemical contact with the underlying gold surface. It is possible to devise methods to overcome this limitation, by controlling the environment of the liquid, either by depositing in an inert environment or by encapsulating the liquid metal directly during deposition. This should open up new venues for metal liquid nanocapsules ${ }^{27,28}$.

\section{Conclusions}

This work demonstrates the importance of controlling the balance between surface tension and surface adhesion in pattern formation of liquid metal at the micron range. In all the conducted experiments, with all the different liquid metals used in this study on either gold or silicon oxide substrates, one common feature was observed, namely that as the liquid metal was extruded through the pore, it expanded well beyond the dimensions of the pore. Furthermore, in the case of $\mathrm{SiO}_{2}$, there was no chemical reaction between the liquid metal with the underlying surface. As the metal was extruded, an oxide film began to form and the metal line consisted of connected blobs of metal, created by spontaneous droplet formation. In this case, we may distinguish between the dynamic deposition of the initial line, and the stable printed pattern that is formed spontaneously afterwards, with characteristic widths of 1-3 $\mu \mathrm{m}$, and $10-15 \mu \mathrm{m}$, respectively. In the case of gold-coated $\mathrm{SiO}_{2}$, the alloying reactions-so-called reactive wetting-were sufficient to prevent droplet formation, and instead favored the formation of a continuous line, albeit wider due to diffusive spreading. The considerably wider pattern-more than two orders-of-magnitude greater than the size of the pore-could be attributed to the strong contact between the glass tip and the underlying substrate. This pattern, too, proved unstable for thick gold films ( $>40 \mathrm{~nm}$ ) due to reactive wetting which ultimately altered its shape. Nonetheless, it may still be possible to reduce the width by controlling the tip-substrate distance, and depositing the metal at a slightly elevated position above the substrate, whereas the extent of the reactivity could be controlled by depositing on thin gold films $(\sim 10 \mathrm{~nm})$. By controlling 
both the distance to and the thickness of the gold film, we estimate that it would be possible to achieve stable patterns with sub-micron width on gold-coated substrates.

\section{Methods}

Eutectic GaIn was purchased from Sigma Aldrich. Gallium Alloys, GaInZn (67:29:4\%) and GaInSn (62:25:13\%), were purchased from Kojundo Chemical Laboratory Co, Ltd., Japan. Solvents were purchased from Wako Pure Chemicals, Japan. Glass capillaries (with filament, Cat. No. GD-1) with a nominal O.D. of $1 \mathrm{~mm}$, and length of $9 \mathrm{~cm}$, were purchased from Narishige Co. Gold electroless plating was performed following the recipes of Jin et al. ${ }^{24}$ and $\mathrm{Hu}$ et al. ${ }^{23}$ with several modifications. For cleaning and surface hydroxylation, the glass tubes were immersed in a freshly made piranha solution $\left(7: 3:: \mathrm{H}_{2} \mathrm{SO}_{4}: \mathrm{H}_{2} \mathrm{O}_{2}\right)$ for 30-60 min in a Teflon bath, rinsed thoroughly with triple-distilled water (TWD) and dried with a flow of $\mathrm{N}_{2}$ gas. For surface functionalization of the inner surface of the tubes, a solution of 9.1\% APTMS in methanol was directly applied by connecting the capillaries to a syringe pump (either Harvard Apparatus Co., Model Pump 11 or YMC Co., Model YSP-201). The tubes were pumped 3-4 times, after which the free end was sealed with parafilm and left completely full overnight (16-24 h). The tubes were then cleaned by pumping methanol followed by TDW and dried with $\mathrm{N}_{2}$ gas. A solution of $10 \mathrm{~nm}$ Gold Nanoparticles (GNPs) in water (COSMO Bio Co., Cat. No. UG-10-20) was used to form the self-assembled monolayers of GNPs on the inner surface of the glass capillary, by pumping the dry functionalized tubes with the GNP solution 3-4 times, before sealing the free end and leaving it for 3-4 h. The electroless deposition was activated by introducing a solution of $0.5-1 \%$ Hydrogen tetrachloroaurate (III) hydrate ( $99.8 \%$, STREM Chemical Inc.) and 5\% $\mathrm{H}_{2} \mathrm{O}_{2}$ (50 wt\%, Sigma-Aldrich Cat. No. 516813). The reaction proceeded as soon as the solution reached the cavity. After 20 min of slow pumping, the tubes were cleaned with TDW and dried with $\mathrm{N}_{2}$ gas. Water for all solutions was filtered through a $0.02 \mu \mathrm{m}$ syringe filter (Whatman 6809-2102 Anotop 25). The quality of the long-term adhesion of the gold film to the glass was noted in two separate instances: one, in which several coated tubes were sonicated in TDW with the coating remaining intact, and the second time, when several tubes were left for 2-3 months inside a closed box, and the coating appeared intact.

P-type boron-doped silicon substrates with $300 \mathrm{~nm}$ oxide were purchased from the Electronics and Materials Co., Japan. Gold substrates were prepared by thermal vapor deposition (TVD) of Cr ( $5 \mathrm{~nm})$ followed by $\mathrm{Au}(\sim 100 \mathrm{~nm})$ on clean hydroxylated silicon substrates, producing a mean roughness of $\sim 0.5 \mathrm{~nm}$ for smooth printing.

Two substrates were prepared for Energy Dispersive X-ray spectroscopy (EDS) measurements by TVD of $\mathrm{Au}(10 \mathrm{~nm}) / \mathrm{Cr}(5 \mathrm{~nm}) / \mathrm{SiO}_{2}$ and $\mathrm{Au}(40 \mathrm{~nm}) / \mathrm{Cr}(5 \mathrm{~nm}) / \mathrm{SiO}_{2}$. The EGaIn was patterned on these substrates by taking a very small portion of EGaIn and using ceramic tweezers to draw a line by gently and quickly sliding the tweezer on top of the Au substrate. All samples were stored in ambient conditions.

Pipettes were formed in a thermal puller, PC-100 (Narishige Co.), using one step at a nominal operating power of $61 \mathrm{~W}$ at full load. Prior to filling the pipette, diluted $\mathrm{HCl}$ was added to the liquid metal to remove the oxide. Excess $\mathrm{HCl}$ was then collected and removed. Initial filling of the pipette consisted of two steps: (1) a Hamilton syringe (\#1705, $33 \mathrm{ga}$ ) introduced the metal liquid into the pipette; (2) the pipette was then placed inside a centrifuge (mini centrifuge, Model MF-12000, AS ONE Co.), for $90 \mathrm{~s}$ at $2000 \mathrm{rpm}$. The filled pipette was then placed inside a home-made motorized stage (Sigma Koki Co., Model SG SP 26-50) and pipette holder, controlled by PC with a dedicated LabVIEW program. Line patterning was monitored with a side camera (TOSHIBA, JK-TUS3H) and a top camera (SHODENSHA, CI500CU and HIROX, CX-7575CS), and was recorded for the duration of the experiment. Pressure was applied to the pipette using an HPLC pump, LC-10AD (Shimadzu Co.). Optical imaging of the substrate after deposition was performed using an Olympus BX51M microscope, operating in Bright Field or Dark Field modes. SEM imaging and EDS measurements were done in JSM-7600F (JOEL). EDS measurements were performed immediately $(\sim 1 \mathrm{~h})$ after sample preparation and 1 week later. Acceleration voltage was $15 \mathrm{kV}$.

\section{Data availability}

Supplementary Information is available from Nature website.

Received: 28 October 2020; Accepted: 11 February 2021

Published online: 25 February 2021

\section{References}

1. Boley, J. W., White, E. L., Chiu, G. T.-C. \& Kramer, R. K. Direct writing of gallium-indium alloy for stretchable electronics. Adv. Funct. Mater. 24. https://doi.org/10.1002/adfm.201303220 (2014).

2. Yoon, Y., Kim, S., Kim, D., Kauh, S. K. \& Lee, J. Four degrees-of-freedom direct writing of liquid metal patterns on uneven surfaces. Adv. Mater. Technol. https://doi.org/10.1002/admt.201800379 (2018).

3. Votzke, C., Daalkhaijav, U., Mengüç, Y. \& Johnston, M. L. 3D-Printed liquid metal interconnects for stretchable electronics. IEEE Sens. J. 19, 3832-3840. https://doi.org/10.1109/JSEN.2019.2894405 (2019).

4. Dickey, M. D. Emerging applications of liquid metals featuring surface oxides. ACS Appl. Mater. Interfaces. 6, 18369-18379. https ://doi.org/10.1021/am5043017 (2014).

5. Ladd, C., So, J.-H., Muth, J. \& Dickey, M. D. 3D printing of free standing liquid metal microstructures. Adv. Mater. 25, 5081-5085. https://doi.org/10.1002/adma.201301400 (2013).

6. Chiechi, R. C., Weiss, E. A., Dickey, M. D. \& Whitesides, G. M. Eutectic gallium-indium (EGaIn): A moldable liquid metal for electrical characterization of self-assembled monolayers. Angew. Chem. Int Ed Engl 47, 142-144. https://doi.org/10.1002/anie.20070 3642 (2008)

7. Khondoker, M. A. H. \& Sameoto, D. Fabrication methods and applications of microstructured gallium based liquid metal alloys. Smart Mater. Struct. 25. https://doi.org/10.1088/0964-1726/25/9/093001 (2016).

8. Kim, S., Oh, J., Jeong, D. \& Bae, J. Direct wiring of eutectic gallium-indium to a metal electrode for soft sensor systems. ACS Appl. Mater. Interfaces. 11, 20557-20565 (2019). 
9. Park, Y.-G., An, H. S., Kim, J.-Y. \& Park, J.-U. High-resolution, reconfigurable printing of liquid metals with three-dimensional structures. Sci. Adv. 5 (2019).

10. Park, Y.-G. et al. Three-dimensional, high-resolution printing of carbon nanotube/liquid metal composites with mechanical and electrical reinforcement. Nano Lett. 19, 4866-4873 (2019).

11. Zhao, X., Xu, S. \& Liu, J. Surface tension of liquid metal: Role, mechanism and application. Front. Energy 11, 535-567. https://doi. org/10.1007/s11708-017-0463-9 (2017).

12. Ding, Y., Zeng, M. \& Fu, L. Surface chemistry of gallium-based liquid metals. Matter 3, 1477-1506 (2020).

13. Shin, G., Jeon, B. \& Park, Y.-L. Direct printing of Sub-30 $\mu \mathrm{m}$ liquid metal patterns on three-dimensional surfaces for stretchable electronics. J. Micromech. Microeng 30. https://doi.org/10.1088/1361-6439/ab6dbc (2020).

14. Bao, J., Otsuka, Y., Etoh, R., Usami, Y. \& Matsumoto, T. Local-field-induced current noise in shape-limited self-doped polyaniline. Nanotechnology 31, 365203. https://doi.org/10.1088/1361-6528/ab96e4 (2020).

15. Diebold, A. V. et al. Electrowetting-actuated liquid metal for RF applications. J. Micromech. Microeng. 27 (2017).

16. 16Iwata, F., Nagami, S., Sumiya, Y. \& Sasaki, A. Nanometre-scale deposition of colloidal Au particles using electrophoresis in a nanopipette probe. Nanotechnology 18 (2007).

17. Salancon, E. \& Tinland, B. Filling nanopipettes with apertures smaller than $50 \mathrm{~nm}$ : dynamic microdistillation. Beilstein J. Nanotechnol. 9, 2181-2187. https://doi.org/10.3762/bjnano.9.204 (2018).

18. Yazdanpanah, M. M., Harfenist, S. A. \& Cohn, R. W. Gallium-driven assembly of gold nanowire networks. Appl. Phys. Lett. 85 (2004).

19. Zhao, W. et al. Single-fluxon controlled resistance switching in centimeter-long superconducting gallium-indium eutectic nanowires. Nano Lett. 15, 153-158 (2015).

20. Kim, H.-J., Son, C. \& Ziaie, B. A multiaxial stretchable interconnect using liquid-alloy-filled elastomeric microchannels. Appl. Phys. Lett. 92. https://doi.org/10.1063/1.2829595 (2008).

21. Hirsch, A., Michaud, H. O., Gerratt, A. P., de Mulatier, S. \& Lacour, S. P. Intrinsically stretchable biphasic (solid-liquid) thin metal films. Adv. Mater. 28, 4507-4512. https://doi.org/10.1002/adma.201506234 (2016).

22. Yazdanpanah, M. M. Near Room Temperature Self-Assembly of Nanostructures by reactions of Gallium with metal thin films $\mathrm{PhD}$ thesis, University of Louisville, (2006).

23. Hu, J., Li, W., Chen, J., Zhang, X. \& Zhao, X. Novel plating solution for electroless deposition of gold film onto glass surface. Surf. Coat. Technol. 202, 2922-2926 (2008).

24. Jin, Y. et al. Controlled nucleation and growth of surface-confined gold nanoparticles on a (3-aminopropyl)trimethoxysilanemodified glass slide: A strategy for SPR substrates. Anal. Chem. 73, 2843-2849 (2001).

25. Doudrick, K. et al. Different shades of oxide: from nanoscale wetting mechanisms to contact printing of gallium-based liquid metals. Langmuir 30, 6867-6877. https://doi.org/10.1021/la5012023 (2014).

26. Chen, Y., Schneider, P. \& Erbe, A. Investigation of native oxide growth on zinc in different atmospheres by spectroscopic ellipsometry. Phys. Status Solidi A 209, 846-853. https://doi.org/10.1002/pssa.201100542 (2012).

27. Lin, Y., Genzer, J. \& Dickey, M. D. Attributes, fabrication, and applications of gallium-based liquid metal particles. Adv. Sci. (Weinh) 7, 2000192. https://doi.org/10.1002/advs.202000192 (2020).

28. Markvicka, E. J., Bartlett, M. D., Huang, X. \& Majidi, C. An autonomously electrically self-healing liquid metal-elastomer composite for robust soft-matter robotics and electronics. Nat. Mater 17, 618-624. https://doi.org/10.1038/s41563-018-0084-7 (2018).

\section{Acknowledgements}

G.I.L. acknowledges the financial support of the Japanese Society for the Promotion of Science for a JSPS Fellowship (2017-2019). The authors are grateful to Prof. Futoshi Iwata and his group in Shizuoka University for preparation of laser-heated nanopipettes and helpful discussions, to Dr. Akihiro Ito of Osaka University for help with SEM imaging and to Ms. Aya Takeuchi for photography. G.I.L. is grateful to Dr. Eduard Mastov for helpful comments on the manuscript.

\section{Author contributions}

G.I.L. conceived the research, designed and performed all the fountain pen lithography experiments in the lab of T. Matsumoto. J.B. and Y.U. provided assistance and guidance on the instrumentation. Y.O. constructed the nanopipette motorized stage with optical monitoring and computer interface. L.S. performed SEM imaging of the samples. T.M. prepared the samples for EDS and performed the EDS measurements with guidance from T. Matsumoto. G.I.L. and T. Matsumoto discussed the results, wrote the manuscript, and all authors reviewed the manuscript.

\section{Competing interests}

The authors declare no competing interests.

\section{Additional information}

Supplementary Information The online version contains supplementary material available at https://doi. org/10.1038/s41598-021-84065-4.

Correspondence and requests for materials should be addressed to G.I.L. or T.M.

Reprints and permissions information is available at www.nature.com/reprints.

Publisher's note Springer Nature remains neutral with regard to jurisdictional claims in published maps and institutional affiliations. 
(c) (i) Open Access This article is licensed under a Creative Commons Attribution 4.0 International cc) License, which permits use, sharing, adaptation, distribution and reproduction in any medium or format, as long as you give appropriate credit to the original author(s) and the source, provide a link to the Creative Commons licence, and indicate if changes were made. The images or other third party material in this article are included in the article's Creative Commons licence, unless indicated otherwise in a credit line to the material. If material is not included in the article's Creative Commons licence and your intended use is not permitted by statutory regulation or exceeds the permitted use, you will need to obtain permission directly from the copyright holder. To view a copy of this licence, visit http://creativecommons.org/licenses/by/4.0/.

(C) The Author(s) 2021 